\title{
Output Feedback Stabilization of Linear PDEs with Finite Dimensional Input-Output Maps and Kelvin-Voigt Damping
}

\author{
Aditya A. Paranjape and Soon-Jo Chung
}

\begin{abstract}
In this paper, we consider systems of partial differential equations with a finite relative degree between the input and the output. In such systems, an output feedback controller can be constructed to regulate the output with the desired convergence properties. Although the zero dynamics are infinite dimensional, we show that the controller alters the boundary conditions in such a way that it leads to a predictable expansion in the stable operating envelope of the system. Moreover, the expansion of the stable envelope depends only on the boundary conditions and the structure of the PDE, and is independent of the system parameters. The methodology is extended to output tracking and time-varying forcing functions as well. The phenomenon investigated in the paper is quite unique to partial differential equations and without any parallel in systems of ODEs.
\end{abstract}

\section{INTRODUCTION}

This paper is concerned with control of systems described by linear second order partial differential equations (PDEs)

$$
\begin{aligned}
& \frac{\partial^{2} w(t, x)}{\partial t^{2}}+\left(\eta \frac{\partial}{\partial t}+1\right) \mathcal{K} w(t, x)=g w(t, x), \\
& y(t)=\int_{0}^{1} w(t, x) d x, 0 \leq x \leq 1, \quad g \in \mathbb{R}
\end{aligned}
$$

equipped with a boundary control $u(t)=\mathcal{U} w(t)$ such that the input-output system is finite dimension and of the form

$\ddot{y}(t)+c \dot{y}(t)+k y(t)=c_{u} \dot{u}(t)+k_{u} u(t)+f\left(\mathbf{w}_{0}(t), \mathbf{w}_{1}(t), y(t)\right)$

for some constants $c, k, c_{u}$ and $k_{u}$. Here, $\mathbf{w}_{0}(t)=$ $\left[w(t, 0), w_{x}(t, 0), \ldots\right]$ and likewise for $\mathbf{w}_{1}(t)$, where $w_{x}$ denotes the partial derivative with respect to $x$.

The finite dimensional input-output (FDIO) map makes it tempting to design an output regulator using just output feedback, which reduces the burden on sensing while providing excellent convergence properties at the output. It is also evident that the zero dynamics left behind in the process are infinite dimensional. An analogy with high order systems of ordinary differential equations (ODEs) would lead one to predict trouble in the zero dynamics if left unattended. However, we show in this paper that for systems of PDEs such as (1), the stability of the zero dynamics is actually enhanced because of the output regulator.

The system in (1) and (2) is a coupled PDE-ODE system. Control problems on coupled PDE-ODE systems addressed

Aditya A. Paranjape is with the Department of Aerospace Engineering, Indian Institute of Technology Bombay, Mumbai, India. Member, IEEE. paranjape@aero.iitb.ac.in.

Soon-Jo Chung is with the Department of Aerospace Engineering and Coordinated Science Lab, University of Illinois at Urbana-Champaign, Urbana, IL 61801. Senior Member, IEEE. sjchung@illinois.edu. in the literature view the ODE as a source of external forcing for the PDE [10], [12]. Output feedback problems for PDEs (systems with as well as without uncertainties) have been addressed largely using observers [8], [11], [13]. This paper differs from these in that the controller is an observer-free law based solely on the FDIO map, assuming that the system is fully known. Our emphasis is on proving the stability of the closed-loop system rather than control design itself.

Systems with FDIO maps do appear in practice, and most notably in flexible wings on aircraft [9]. Aircraft wings are susceptible to an oscillatory instability at high speeds, called flutter. Flutter mitigation strategies have evolved in the recent years to use trailing edge flaps for suppressing vibrations [1], [2]. The resulting control problem involves the stabilization of the coupled bending-torsion dynamics. The torsion dynamics, incidentally, are identical to (1), as shown by the authors [9]. Based on basic aeroelastic models of flutter (see Chapter 5 in [6]), there is enough reason to believe that stabilizing the torsion dynamics using the FDIO logic may increase the flutter speed substantially through stiffness injection in the torsion dynamics. Separately, an FDIO formulation such as this may also be used when it is possible to select outputs as part of control design. The benefit of an FDIO map is that it allows us to use any of a large number of finite dimensional control techniques for infinite dimensional systems. On the other hand, the "zero dynamics" left behind are infinite dimensional and need to be analysed for stability.

It is unreasonable to expect a system such as (1) to be stabilized for all values of $g$ using just input-output control found from Eq. (2). Rather, the best we can hope for is a predictable increase in the range of stable values of $g$ : this is what we mean in this paper by "growth of the stable operating envelope," since the parameter $g$ typically depends on the operating conditions of the system. The objective of this paper is to prove the growth in the stable oper ating envelope for a class of systems described by linear partial differential equations. While the expansion of the stable envelope may seem a rather modest objective, it is in fact of considerable utility in practical settings where the range of operations is restricted for other reasons. As an example, it suffices to simply increase the flutter speed beyond the normal operating envelope (decided by performance requirements) rather than making the aircraft flutter-resilient at all possible air speeds.

The paper is organized as follows. In Section II, the problem formulation is made precise, including the identification of the class of PDEs to which it applies. In Section III, we derive the conditions under which the closed-loop is stable. 
In Section IV, we estimate the asymptotic eigenvalues to explain the mechanism behind the expansion in the stable operating envelope. We also identity possibly problematic boundary conditions. The method is illustrated for an EulerBernoulli beam in Section V, and extended to output tracking and time-varying forcing functions in Section VI.

\section{Problem Formulation}

We consider systems described by a second order PDE of the form

$$
\begin{aligned}
& w_{t t}(t, x)+\left(\eta \frac{\partial}{\partial t}+1\right) \mathcal{K} w(t, x)=g w(t, x), \\
& y(t)=\int_{0}^{1} w(t, x) d x, \mathcal{N} w(t)=0 \\
& u(t)=\mathcal{U} w(t)=\int_{0}^{1}(\mathcal{K} w)(t, x) d x+f\left(\mathbf{w}_{0}, \mathbf{w}_{1}, y\right)
\end{aligned}
$$

for some function $f(\cdot)$, and where $w_{t t}=\frac{\partial^{2} w}{\partial t^{2}}$. The constant $\eta>0$ is the Kelvin-Voigt damping coefficient, while $g \in \mathbb{R}$ is the coefficient of the external forcing term. The operator $\mathcal{K}$ is purely a spatial operator; i.e., independent of time. Equation (3) is representative of a wide variety of physical systems such as torsion, bending and axial deformation of elastic structures, thermal conduction, mass transport phenomena, etc. The domain of $\mathcal{K}, D(\mathcal{K}) \subseteq Z$, where $Z$ is the standard Hilbert space. Recall that $\mathbf{w}_{0}=\left[w(t, 0), w_{x}(t, 0), \ldots\right]$ and $\mathbf{w}_{1}=\left[w(t, 1), w_{x}(t, 1), \ldots\right]$ is defined likewise. Note that only as many derivatives are needed in $\mathbf{w}$ as the highest spatial derivative in $\mathcal{K}$.

Differentiating the output twice yields

$$
\ddot{y}(t)=g y(t)-\eta \dot{u}(t)-u(t)+\left(\eta \frac{\partial}{\partial t}+1\right) f\left(\mathbf{w}_{0}, \mathbf{w}_{1}, y\right)
$$

Since we assume that all quantities on the boundary $x=0$ and $x=1$ can be measured, it follows that one can design $u(t)$ readily to ensure that the output dynamics satisfy

$$
\ddot{y}+c_{u} \dot{y}+k_{u} y=0
$$

for constants $c_{u}, k_{u}>0$ which are chosen to achieve any desired output response. However, the zero dynamics left behind are infinite dimensional and not guaranteed to be stable in general.

Assumption 1: The operator

$$
\mathcal{A}=\left[\begin{array}{cc}
0 & 1 \\
-\mathcal{K}+g & -\eta \mathcal{K}
\end{array}\right]
$$

is a Riesz spectral operator, and has simple eigenvalues for almost all $g$.

Let $\left\langle z_{1}, z_{2}\right\rangle$ denote the usual inner product for $z_{1}, z_{2} \in Z$, and let $\|z\|$ denote the standard $\mathcal{L}_{2}$ norm for $z \in Z$. Note that this is a spatial norm. For an operator $\mathcal{T}: Z \rightarrow Z$, we denote the induced norm by $\|\mathcal{T}\|_{i}$.

Assumption 2: The operator $\mathcal{A}$ is the infinitesimal generator of a uniform exponentially stable semi-group $T_{o}(t)$ for $g<g_{\mathrm{OL}}$ and for the boundary conditions $\mathcal{N} w=\mathcal{U} w=0$. Furthermore, when $g>g_{\mathrm{OL}}$, the operator $\mathcal{A}$ is unstable.
We call $g_{\mathrm{OL}}$ the stable envelope of the open-loop system and define a similar quantity $g_{\mathrm{CL}}$ for the closed-loop system (3) and (4).

Problem Statement: determine $g_{\mathrm{CL}}$ as a function of $g_{\mathrm{OL}}$ and $\mathcal{K}$.

\section{Stability ANALYSis}

We define a new coordinate $v(t, x)=\int_{0}^{x} w(t, z) d z$. First, we note that the $v(t, 1)=\int_{0}^{1} w(t, z) d z=y(t)$. The finite dimensional controller from (4) ensures that the output is exponentially converging (see (6)). We get

$$
\begin{aligned}
v(t, 1)=\mu(t)= & e^{-0.5 c_{u} t}\left(v(0,1) \cos \left(\omega_{d} t\right)\right. \\
& \left.+\frac{v_{t}(0,1)+0.5 c_{u} v(0,1)}{\omega_{d}} \sin \left(\omega_{d} t\right)\right)(7)
\end{aligned}
$$

where $\omega_{d}=\sqrt{k_{u}-c_{u}^{2} / 4}$. The symbol $\mu(t)$ has been introduced to denote the behavior of the output under feedback. In other words, $y(t)=\mu(t)$ under feedback. The dynamics of $v$ are thus given by

$$
\begin{aligned}
& v_{t t}(t, x)+\left(\eta \frac{\partial}{\partial t}+1\right) \mathcal{K} v(t, x)=g v(t, x) \\
& +\left(\eta \frac{\partial}{\partial t}+1\right)(\mathcal{K} v)(t, 0) \\
& \mathcal{N} v_{x}=0, v(t, 0)=0, v(t, 1)=\mu(t)
\end{aligned}
$$

The boundary condition $v(t, 1)$ is exponentially decaying. In order to prove the stability of (8), we will first show the stability of the system

$$
\begin{aligned}
& v_{t t}+\left(\eta \frac{\partial}{\partial t}+1\right) \mathcal{K} v=g v+\left(\eta \frac{\partial}{\partial t}+1\right)(\mathcal{K} v)(t, 0) \\
& \mathcal{N} v_{x}=0, v(t, 0)=v(t, 1)=0
\end{aligned}
$$

Notice that $v(t, 1)$ has been set to 0 in (9). Thus, (9) can be viewed as the unperturbed dynamics corresponding (8) which are perturbed at the boundary $x=1$ by the exponentially decaying $\mu(t)$.

\section{A. Stability of (9)}

Consider the system (3) with $\mathcal{N} w=\mathcal{U} w=0$. Using separation of variables, the solution to (3) can be cast into the form $w(t, x)=\gamma_{o}(t) \phi_{o}(x)$. This gives

$$
\frac{\ddot{\gamma}_{o}(t)-g \gamma_{o}(t)}{\eta \dot{\gamma}_{o}(t)+\gamma_{o}(t)}=-\frac{\mathcal{K} \phi_{o}(x)}{\phi_{o}(x)}=-\lambda_{\mathrm{OL}}^{p}
$$

where $p$ denotes the number of boundary conditions in $\mathcal{N}$ and $\mathcal{U}$. Recall that the principal eigenvalue, $\lambda_{\mathrm{OL}, 1}$, is found by solving for $\phi(x)$ with the boundary conditions $\mathcal{N} \phi=\mathcal{U} \phi=0$.

We define the variable separation $v(t, x)=\gamma_{c}(t) \phi(x)$ in (9) and a new operator $\tilde{\mathcal{K}}: \tilde{\mathcal{K}} v(t, x)=(\mathcal{K} v)(t, x)-$ $(\mathcal{K} v)(t, 0)$. The closed loop dynamics (9) give rise to the following equation:

$$
\frac{\ddot{\gamma}_{c}(t)-g \gamma_{c}(t)}{\eta \dot{\gamma}_{c}(t)+\gamma_{c}(t)}=-\frac{\tilde{\mathcal{K}} \phi(x)}{\phi(x)}=-\lambda_{\mathrm{CL}}^{p}
$$


The boundary conditions in (9) can be imposed on the eigenfunction $\phi(x)$ to obtain the principal eigenvalue $\lambda_{c, 1}$ for the closed-loop system.

Proposition 1: The stable envelope of the closed-loop system is related to that of the open-loop system via the equation

$$
g_{\mathrm{CL}}=\left(\frac{\lambda_{\mathrm{CL}, 1}}{\lambda_{\mathrm{OL}, 1}}\right)^{p} g_{\mathrm{OL}}
$$

Proof: The dynamics of $\gamma_{o}(t)$ in (10) are given by $\ddot{\gamma}_{o}+$ $\lambda^{p} \eta \dot{\gamma}_{o}+\left(\lambda^{p}-g\right) \gamma_{o}=0$. The stable envelope is thus $\lambda_{\mathrm{OL}, 1}^{p}$ for the open loop. The stable envelope of the closed-loop system is determined likewise.

\section{B. Riesz-Spectral Properties of (9) for $g<g_{\mathrm{CL}}$}

The system in (9) can be recast into the vector form

$$
\dot{\bar{v}}=\mathcal{A}_{c} \bar{v}
$$

where $\bar{v}=\left[v, v_{t}\right]^{\top}$, and the operator $\mathcal{A}_{c}$ is given by

$$
\mathcal{A}_{c}=\left[\begin{array}{cc}
0 & 1 \\
-\tilde{\mathcal{K}}+g & -\eta \tilde{\mathcal{K}}
\end{array}\right]
$$

It can be checked, from Corollary 2.3.6 in [3], that $\mathcal{A}_{c}$ is also Riesz-spectral. Moreover, its eigenvalues are simple for almost all $g$. Note that $\mathcal{A}_{c}$ is stable when $g<g_{\mathrm{CL}}$. Let $-\beta_{k}, k \in \mathbb{N}$, denote the eigenvalues of $\mathcal{A}_{c}$. We assume that $\operatorname{Re}\left(\beta_{k}\right) \leq-k \alpha(g)$ for some constant $\alpha(g)>0$ for $g<g_{\mathrm{CL}}$. This assumption is true for a large class of boundary value problems of the form (11).

Let $T(t)$ denote the semi-group generated by $\mathcal{A}_{c}$. The semi-group is exponentially stable; i.e., there exist constants $M(g), \omega(g) \in \mathbb{R}^{+}$when $g<g_{\text {closed }}$ such that $\|T(t)\|_{i} \leq$ $M(g) e^{-\omega(g) t}$. The exponential bound $\omega(g)=\inf \left|\operatorname{Re}\left(\beta_{k}\right)\right|$.

Since $\mathcal{A}_{c}$ is Riesz-spectral with simple eigenvalues, there exist Riesz basis functions $\psi_{i}(x)$ and $\theta_{i}(x), i \in \mathbb{N}$, satisfying $\left\langle\psi_{i}, \theta_{j}\right\rangle=\delta_{i j}$, such that

$$
\begin{aligned}
& h(t, x)=\sum_{n \in \mathbb{N}}\left\langle h(t), \theta_{n}\right\rangle \psi_{n}(x)=\sum_{n \in \mathbb{N}}\left\langle h(t), \psi_{n}\right\rangle \theta_{n}(x), \\
& T(t) \cdot=\sum_{n \in \mathbb{N}} e^{-\beta_{n} t}\left\langle\cdot, \psi_{n}\right\rangle \theta_{n}(x)
\end{aligned}
$$

Assumption 3: The derivative of $\theta_{n}(x)$ exists for all $x$ and $n$, and is given by $\theta_{n, x}(x)=\sum_{i} n \alpha_{n, i} \theta_{n}(x)$ for some constants $\alpha_{n, i}$ which satisfy $\left|\alpha_{n, n}\right| \leq \tilde{\alpha}$ for all $n$ and some constant $\tilde{\alpha}>0$.

Assumption 3 is, in fact, quite weak. Since $\theta_{n}(x)$ forms a basis in $Z$, it follows that series expansion always exists. The bounds on the coefficients are a consequence of (11).

Proposition 2: Let $p(t) \in Z$ be given by $p(t, x)=$ $\sum_{n \in \mathbb{N}} p_{n}(t) \theta_{n}(x)$, where $p_{n}(t)=(l-n)^{-1} e^{-\kappa_{n} t}\left\langle A, \psi_{n}\right\rangle$, where $0<\kappa \leq \kappa_{n}$ for all $n ; l \in \mathbb{R}$, and $\|A\|$ is bounded. Then, both $\|p(t)\|$ and $\left\|p_{x}(t)\right\|$ decay to zero exponentially.

Proof: It follows from the properties of the Riesz basis that there exists a constant $m$ such that $\|p(t)\|^{2} \leq$ $m^{2} \sum_{k}\left|\left\langle p(t), \psi_{n}\right\rangle\right|^{2}$. Note that

$$
\left|\left\langle p(t), \psi_{n}\right\rangle\right|^{2}=\frac{e^{-2 \kappa_{n} t}}{|l-n|^{2}}\left|\left\langle A, \psi_{n}\right\rangle\right|^{2} \leq e^{-2 \kappa t} m^{2} M^{2}\|A\|^{2} .
$$

for some constant $M>0$. The last inequality is also a property of the Riesz basis. Thus, $\|p(t)\| \leq e^{-\kappa t} m M\|A\|$, which proves that $\|p(t)\|$ decays to zero exponentially.

We start with $p_{x}(t)=\sum_{n \in \mathbb{N}} p_{n}(t) \theta_{n, x}(x)$. Since $\theta_{n}(x)$ is the basis for $Z$, we can write $\theta_{n, x}(x)=\sum_{i} n \alpha_{n, i} \theta_{i}(x)$ for some constants $\alpha_{n, i}$. Thus,

$$
\begin{aligned}
\left\langle p_{x}(t), \psi_{n}\right\rangle & =\sum_{n} p_{n}(t) n \alpha_{n, n} \\
\left\|p_{x}(t)\right\|^{2} & \leq \tilde{M}^{2}\left|\left\langle p_{x}(t), \psi_{n}\right\rangle\right|^{2} \\
& \leq \tilde{M}^{2} e^{-2 \kappa t} \sum_{n} \frac{n^{2}\left|\left\langle A, \psi_{n}\right\rangle\right|^{2}}{(n-l)^{2}} \alpha_{n, n}^{2}
\end{aligned}
$$

where $\quad \tilde{M}$ is a constant. Thus, $\left\|p_{x}(t)\right\| \leq$ $e^{-\kappa t} \tilde{M} \tilde{\alpha} \sqrt{\sum_{n} \frac{n^{2}\left|\left\langle A, \psi_{n}\right\rangle\right|^{2}}{(n-l)^{2}}}$. Since $n^{2} /(l-n)^{2}$ converges to unity at a geometric rate, we deduce that the sum on the right hand side converges, and $\left\|p_{x}(t)\right\| \rightarrow 0$ exponentially fast.

\section{Stability of (8) and (3)}

Next, we convert the system in (8) into one with homogeneous boundary conditions. We choose a continuously differentiable function $q(x) \in D(\mathcal{K})$ which satisfies the boundary conditions $\mathcal{N}\left(q_{x}\right)=0, q(0)=0$ and $q(1)=1$. Finally, we introduce a new variable $\tilde{v}(t, x)=v(t, x)-q(x) \mu(t)$ in (8), so that it can be cast into the form

$\tilde{v}_{t t}+\left(\eta \frac{\partial}{\partial t}+1\right) \mathcal{K} \tilde{v}=g \tilde{v}+\left(\eta \frac{\partial}{\partial t}+1\right)(\mathcal{K} \tilde{v})(t, 0)$ $+q(x)(\ddot{\mu}(t)-g \mu(t))+((\mathcal{K} q)(x)-(\mathcal{K} q)(0))(\eta \dot{\mu}(t)-g \mu(t))$

$\mathcal{N} \tilde{v}_{x}=0, \tilde{v}(t, 0)=\tilde{v}(t, 1)=0$

The system in (15) is similar to (9), except that it is perturbed by terms in $\mu(t)$ that decay exponentially in time.

To simplify the notation, we designate the right hand side of (15) by $\rho(t, x)$. Next, we write $[0, \rho(t, x)]$ as

$$
[0, \rho(t, x)]^{\top}=A_{1}(x) e^{-\nu_{1} t}+A_{2}(x) e^{-\nu_{2} t}
$$

where $\nu_{1}=c_{u}+i \omega_{d}$ and $\nu_{2}=c_{u}-i \omega_{d}$. The functions $A_{1}(x)$ and $A_{2}(x)$ can be found by direct analogy with Eq. (15).

Theorem 1: The system (15) is exponentially stable if $g<g_{\mathrm{CL}}$. Moreover, the norm of system state, $\|w(t)\| \rightarrow 0$ exponentially fast.

Proof: Using the variation-of-constants formula, it follows that

$$
\overline{\tilde{v}}(t, x)=T(t) \overline{\tilde{v}}(0, x)+\int_{0}^{t} T(t-s)[0, \rho(s)]^{\top} d s
$$

where $\overline{\tilde{v}}=\left[\tilde{v}, \tilde{v}_{t}\right]^{\mathrm{T}}$. It is clear that $\|T(t) \tilde{v}(0)\| \rightarrow 0$ exponentially fast. Therefore, only the second term needs to be analysed further.

For now, we confine our analysis to the $A_{1}(x) e^{-\nu_{1} t}$, since the analysis for the second term would be identical. We will show that

$$
h(t, x)=\int_{0}^{t} T(t-s) A_{1}(x) e^{-\nu_{1} s} d s
$$


converges to zero exponentially fast, from which it follows that $\tilde{v}(t)$ also converges to zero exponentially.

From Eq. (14), we get

$$
\begin{aligned}
& T(t-s) A_{1}(x) e^{-\nu_{1} s} \\
& =\sum_{n \in \mathbb{N}} e^{-\beta_{n}(t-s)}\left\langle A_{1}(x) e^{-\nu_{1} s}, \psi_{n}(x)\right\rangle \theta_{n}(x) \\
& =e^{-\beta_{n} t} \sum_{n \in \mathbb{N}} e^{-\left(\nu_{1}-\beta_{n}\right) s}\left\langle A_{1}(x), \psi_{n}(x)\right\rangle \theta_{n}(x)
\end{aligned}
$$

Substituting into (17)

$$
h(t, x)=\sum_{n}\left(\frac{e^{-\beta_{n} t}-e^{-\nu_{1} t}}{\nu_{1}-\beta_{n}}\right)\left\langle A_{1}(x), \psi_{n}(x)\right\rangle \theta_{n}(x)
$$

It is easy to verify that there exists a constants $m_{0}, m_{1}>0$ such that $\sup _{n}\left|\frac{e^{-\beta_{n} t}-e^{-\nu_{1} t}}{\nu_{1}-\beta_{n}}\right| \leq m_{0} e^{-m_{1} t}$ if $\nu_{1} \neq \beta_{k}$ for any $k \in \mathbb{N}$. If $\nu_{1}=\beta_{k}$ for some $k$, then the integration

$$
\begin{aligned}
& \int_{0}^{t} e^{-\beta_{k} t} e^{-\left(\nu_{1}-\beta_{k}\right) s}\left\langle A_{1}(x), \psi_{k}(x)\right\rangle \theta_{k}(x) \\
& =e^{-\beta_{k} t}\left\langle A_{1}(x), \psi_{k}(x)\right\rangle \theta_{k}(x) t
\end{aligned}
$$

The right hand side clearly decays to 0 exponentially with a rate $e^{-R e\left(\beta_{k}\right) t}$. Therefore, we deduce that there exist constants $m_{0}$ and $m_{1}$ such that $\sup _{n}\left|\frac{e^{-\beta_{n} t}-e^{-\nu_{1} t}}{\nu_{1}-\beta_{n}}\right| \leq$ $m_{0} e^{-m_{1} t} \max \{1, t\}$ (the case of $\nu_{1}=\beta_{k}$ for some $k$ should be understood in the light of (18)). Moreover, since $\beta_{n} \sim \mathcal{O}(n)$ (see Sec. III-B), it follows from Proposition 2 that $\|\tilde{v}(t)\|$ and $\left\|\tilde{v}_{x}(t)\right\|$ decay to zero exponentially.

Next, note that $v(t, x)=\tilde{v}(t, x)+q(x) \mu(t)$, where $q(x)$ is a continuously differentiable function over a compact domain. Thus, it follows that $\|v(t)\|$ and $\left\|v_{x}(t)\right\|$ decay to zero exponentially fast. But $v(t, x)=\int_{0}^{x} w(t, z) d z \Longrightarrow$ $v_{x}(t, \cdot)=w(t, \cdot)$. Thus, even $\|w(t)\| \rightarrow 0$ exponentially.

In fact, since $\|v(t)\|$ and $\left\|v_{x}(t)\right\|$ decay exponentially fast, it follows that $v(t, x) \rightarrow 0$ exponentially fast for each $x$. Since $w(t, x)$ is expected to be continuously differentiable almost everywhere by the virtue of being in $\mathcal{D}(\mathcal{K})$, it follows that $w(t, x) \rightarrow 0$ for all $x$.

\section{Eigenvalues $\lambda_{\mathrm{CL}}$ AND $\lambda_{\mathrm{OL}}$}

The success of the technique relies on the ratio of the principal eigenvalues, $\lambda_{\mathrm{CL}, 1} / \lambda_{\mathrm{OL}, 1}$, being greater than 1 . For mechanical systems, this happens when the open loop system has mixed boundary conditions or purely Neumann boundary conditions. The controller creates two Dirichlet conditions, and flips the other boundary conditions between Neumann and Dirichlet.

The boundary value problem for the closed-loop system is given by

$$
\begin{aligned}
& \mathcal{K} \phi_{c}(x)=\lambda^{p} \phi_{c}(x)+\mathcal{K} \phi_{c}(0), \\
& \mathcal{N} \phi_{c, x}=0, \phi_{c}(0)=\phi_{c}(1)=0
\end{aligned}
$$

Notice that there are $p+1$ boundary conditions. We express the solution $\phi_{c}(x)$ as a linear combination of $p$ basis functions $P_{i}(x ; \lambda)$ (exponential, sinusoidal, hyperbolic sinusoidal), i.e.,

$$
\phi_{c}(x)=\sum A_{i}\left(P_{i}(x ; \lambda)-P_{i}(0 ; \lambda)\right.
$$

Notice that the boundary condition $\phi_{c}(0)=0$ has already been absorbed here. Therefore, the eigenvalues can be found by solving $\mathcal{N} \phi_{c, x}=\phi_{c}(1)=0$.

The boundary value problem for the open loop is along more conventional lines: solve $\mathcal{K} \phi_{o}(x)=\lambda^{p} \phi_{o}(x)$ with $\mathcal{N} \phi_{o}=\mathcal{U} \phi_{o}=0$. The expansion for $\phi_{o}(x)$ is similar to (20), although without $P_{i}(0 ; \lambda)$.

Notice that the problem of determining $\lambda_{\mathrm{OL}}$ and $\lambda_{g c l}$ is a variant of the problem of determining the eigenvalues of the Sturm-Liouville problems. In fact, there is a limited body of results which give the principal eigenvalue as a function of the boundary condition for general Sturm-Liouville operators [5], [4]. Aside frrom the difficulty in extending the results to a general problem $\mathcal{K} \phi(x)=\lambda^{p} \phi(x)$, the closed-loop system has an additional term $(\mathcal{K} \phi)(0)$ which complicates matters.

We focus on the asymptotic case, i.e., where the eigenvalue is of a sufficiently large magnitude. In this case, the only functions that matter for our calculations are sinusoidal: the exponential functions either converge to zero, or diverge along with the hyperbolic sine and cosine. In the latter case, their net contribution to $\phi(x)$ is zero since $\phi(0)=\phi(1)=0$. We consider two sub-cases here.

\section{A. Sinusoidal Eigenfunctions}

When the eigenfunctions include purely sinusoidal terms, the asymptotic eigenvalues of the open loop system can be found by analysing the simpler polynomial

$$
\phi_{o}(x)=A_{1} \sin \left(\lambda_{\mathrm{OL}} x\right)+A_{2} \cos \left(\lambda_{\mathrm{OL}} x\right)
$$

We retain the two boundary conditions from $\mathcal{N}$ and $\mathcal{U}$ with the lowest order, one at each boundary point. The resulting eigenvalues are well-known and of the following form:

- Neumann at both ends or Dirichlet at both ends: $n \pi$. Notice that $n \gg 1$ in the asymptotic case.

- Mixed boundary conditions: $(2 n+1) \pi / 2$.

The closed-loop asymptotic polynomial is given by

$$
\phi_{c}(x)=A_{1} \sin \left(\lambda_{\mathrm{CL}} x\right)+A_{2}\left(\cos \left(\lambda_{\mathrm{CL}} x\right)-1\right), \phi_{c}(1)=0
$$

We note at once that $\lambda_{\mathrm{CL}}=2 \pi$ is a solution to the stated boundary condition. Since we discounted $p-2$ terms from the polynomial, we can retain one more "low order" boundary condition at $x=0$. The eigenvalues that result as a function of the boundary condition at $x=0$ are given by

- $\phi_{c, x}(0)=0: \lambda_{\mathrm{CL}, n}=2 n \pi$; derived from Dirichlet condition in the open loop.

- $\phi_{c, x x}(0)=0: \lambda_{\mathrm{CL}, n}=n \pi$; derived from Neumann condition in the open loop.

The asymptotic expression suggests that the expansion of the stability envelope is the largest when the open-loop system has (i) mixed boundary conditions, and (ii) a Dirichlet boundary condition at $x=0$ (i.e., at the non-collocated end with respect to the control input). 


\section{B. Exponential-Sinusoidal Eigenfunctions}

Consider eigenfunctions of the form

$$
\phi_{o}(x)=e^{-\lambda_{\mathrm{OL}} x}\left(A_{1} \sin \left(\lambda_{\mathrm{OL}} x\right)+A_{2} \cos \left(\lambda_{\mathrm{OL}} x\right)\right)
$$

We retain the two boundary conditions from $\mathcal{N}$ and $\mathcal{U}$ with the lowest order, one at each boundary point. The resulting eigenvalues are well-known and of the following form:

- Neumann at both ends or Dirichlet at both ends: $n \pi$. Notice that $n \gg 1$ yet again, in the asymptotic case.

- Mixed boundary conditions: $\pi / 4+n \pi$.

The closed-loop asymptotic polynomial is given by

$$
\begin{aligned}
& \phi_{c}(x)=e^{-\lambda_{\mathrm{CL}} x}\left(A_{1} \sin \left(\lambda x_{c}\right)+A_{2}\left(\cos \left(\lambda_{\mathrm{CL}} x\right)-1\right)\right), \\
& \phi_{c}(1)=0
\end{aligned}
$$

We note that $\lambda_{\mathrm{CL}}=2 \pi$ is a solution for the stated boundary condition. Yet again, we retain a more "low order" boundary condition at $x=0$. The eigenvalue that results is a function of the boundary condition at $x=0$ :

- $\phi_{c, x}(0)=0$ : the asymptotic solutions satisfy $\sin \left(\lambda_{\mathrm{CL}}\right)+$ $\cos \left(\lambda_{\mathrm{CL}}\right)=1$, which includes $\lambda_{\mathrm{CL}, n}=2 \pi$ among others. Clearly, in this case, the asymptotic approximations are inadequate to draw any further conclusions. It is worth noting that the boundary condition in this case is derived from a Dirichlet condition in the open loop.

- $\phi_{c, x x}(0)=0: \lambda_{\mathrm{CL}, n}=2 n \pi$; derived from Neumann condition in the open loop.

The asymptotic expression suggests possible degradation in stability when the open loop non-collocated boundary condition is Dirichlet. On the other hand, when the boundary condition at the non-collocated end is Neumann, we see a two-fold increase in the eigenvalue and, with it, up to $2^{p}$-fold increase in the stable envelope.

\section{Application: Euler-Bernoulli Beam}

An Euler-Bernoulli beam with Kelvin-Voigt damping is governed by the following equation:

$$
\begin{aligned}
& \xi_{t t}(t, x)+E\left(\eta \frac{\partial}{\partial t}+1\right) \xi_{x x x x}(t, x)=g \xi(t, x) \\
& \xi(t, 0)=\xi_{x}(t, 0)=\xi_{x x}(t, 1)=0 \\
& \xi_{x x x}(t, 1)=u(t), \quad \int_{0}^{1} \xi(t, x) d x=y(t)
\end{aligned}
$$

where $\eta>0$ in the Kelvin-Voigt damping coefficient, and $E$ is the product of the Young's modulus and the second moment of area of the beam.

Proposition 3: The system in (21), with $u(t)=0$, is stable if $g<(1.8754)^{4} E$, and it is unstable otherwise.

Proof: Using variable separation $\xi=\phi(x) \alpha(t)$, it can be checked that

$$
\frac{\phi_{x x x x}(x)}{\phi(x)}=\lambda^{4}=-\frac{\ddot{\alpha}(t)-g \alpha(t)}{E(\eta \dot{\alpha}(t)+\alpha(t))}
$$

It is well-known that the smallest eigenvalue of an EulerBernoulli beam with the stated boundary conditions is $\lambda=$ 1.8754 , from which the result follows.
Differentiating the output in Eq. (21) twice gives

$$
\begin{aligned}
& \ddot{y}(t)=\int_{0}^{1} \ddot{\xi} d x=\int_{0}^{1}\left(g \xi-\eta E \xi_{t x x x x}-E \xi_{x x x x}\right) d x \\
& =g y(t)-E\left(\eta u(t)+u(t)-\eta \xi_{t x x x}(t, 0)-\xi_{x x x}(t, 0)\right)
\end{aligned}
$$

The quantities $\xi_{x x x}(t, 0)$ and $\xi_{t x x x}(t, 0)$ (at the noncollocated end) are the only ones that need to be measured to design the following dynamic control law

$$
\begin{aligned}
\eta \dot{u}(t)+u(t)= & \eta \xi_{t x x x}(t, 0)+\xi_{x x x}(t, 0) \\
& +c_{u} \dot{y}(t)+\left(k_{u}+g / E\right) y(t)
\end{aligned}
$$

with $c_{u}, k_{u}>0$. The output dynamics satisfy $\ddot{y}+E c_{u} \dot{y}+$ $E k_{u} y=0$. The constants $c_{u}$ and $k_{u}$ can be chosen so that the dynamics are critically damped with an arbitrarily fast convergence rate.

To analyse the closed-loop system, we define a new variable $v(x) \triangleq v(t, x)=\int_{0}^{x} \xi(t, z) d z$ whose dynamics are found by differentiating twice with respect to time and substituting for the dynamics of $\xi$ :

$$
\begin{aligned}
& v_{t t}(x)+E\left(\eta v_{t x x x x}(x)+v_{x x x x}(x)\right)=g v(x) \\
& +E\left(\eta v_{t x x x x}(0)+v_{x x x x}(0)\right) \\
& v(0)=v(1)=0 \\
& v_{x}(0)=v_{x x}(0)=v_{x x x}(1)=0
\end{aligned}
$$

This system may be viewed as an idealized closed-loop system in which the output error $y(t) \rightarrow 0$ in almost zero time. Once we establish the exponential stability of (23), the stability of the closed-loop system (21) and (22) follows readily along the lines of Thm. 1 .

Proposition 4: The idealized closed-loop system (23) is exponentially stable and moreover, the expansion of the stable envelope is given by $g_{\mathrm{CL}} \approx 126 g_{\mathrm{OL}}$.

Proof: The eigenfunction polynomial can be verified to be given by

$\phi(x)=A(\sin (\lambda x)-\sinh (\lambda x))+B(\cos (\lambda x)+\cosh (\lambda x)-2)$

subject to $\phi_{x x x}(1)=\phi(1)=0$ and for some constants $A$ and $B$. The eigenvalues are found by solving

$$
1+\cos (\lambda) \cosh (\lambda)=\cos (\lambda)+\cosh (\lambda)
$$

which gives the smallest non-trivial eigenvalue as $\lambda=2 \pi$. The stable envelope can thus be checked to have expanded by a factor $(2 \pi / 1.8754)^{4} \approx 126$.

Notice that the factor of 126 does not depend on the system constants $g$ and $E$. It is a function of the boundary conditions alone.

\section{Generalization to Tracking AND TIME-VARYING SYSTEMS}

\section{A. Tracking Controller}

Recall the input-output map from Eq. (5):

$$
\ddot{y}(t)=g y(t)-\eta \dot{u}(t)-u(t)+f\left(\mathbf{w}_{0}, \mathbf{w}_{1}, y\right)
$$

It is quite straight-forward to design a control law $u(t)$ which ensures that the output tracks a twice-differentiable 
reference signal $r(t)$ such that the dynamics of the tracking error $e(t)=y(t)-r(t)$ satisfies $\ddot{e}+c_{u} \dot{e}+k_{u} e=0$ for appropriately chosen constants $c_{u}, k_{u}>0$. In order to prove stability of the closed-loop system, we can repeat the steps from the previous section. First, we construct the system in Eq. (9), except for the modified boundary condition $v(t, 1)=e(0) e^{-0.5 c_{u} t} \cos \left(\omega_{d} t\right)+r(t)$. The change in coordinates to $\tilde{v}$ leads to an equation similar to (15), with the corresponding modification that the right hand side consists only of terms that converge exponentially and terms that depend on $r(t), \dot{r}(t)$ and $\ddot{r}(t)$. In this case, we can prove that the state $\tilde{v}(t, x)$ is bounded for all $x$ and $t$.

We write the $\tilde{v}$ dynamics along the lines of Eq. (15) and (16):

$$
\begin{aligned}
& \tilde{v}_{t t}+\left(\eta \frac{\partial}{\partial t}+1\right) \mathcal{K} \tilde{v}=g \tilde{v}+\left(\eta \frac{\partial}{\partial t}+1\right)(\mathcal{K} \tilde{v})(t, 0)+\rho(t, x, r) \\
& \mathcal{N} \tilde{v}_{x}=0, \tilde{v}(t, 0)=\tilde{v}(t, 1)=0
\end{aligned}
$$

where $\rho(t, x, r)$ is now the sum of the $\rho(t, x)$ in (16) and terms that depend on $r(t)$ and its derivatives. Notice that $\sup _{t}\|\rho(t, r)\|$ is bounded if $\sup _{t}|r(t)|, \sup _{t}|\dot{r}|, \sup _{t}|\ddot{r}(t)|$ are bounded. It follows that

$$
\tilde{v}(t)=T(t) \tilde{v}(0)+\int_{0}^{t} T(t-s)[0, \rho(t, r)]^{\top} d s
$$

Taking the two norm of both sides, we get

$$
\begin{aligned}
& \|\tilde{v}(t)\| \leq M(g)\left(e^{-\omega(g) t}\|\tilde{v}(0)\|\right. \\
& \left.+\left(\sup _{t}\|\rho(t, r)\|\right) \int_{0}^{t} e^{-\omega(g)(t-s)} d s\right) \\
& \leq M(g)\left(e^{-\omega(g) t}\|\tilde{v}(0)\|+\frac{\sup _{t}\|\rho(t, r)\|}{\omega(g)}\right)
\end{aligned}
$$

It is easy to verify that $\|w(t)\|$ is bounded, following the approach taken for Theorem 1. We have thus proved the following result.

Theorem 2: An output feedback tracking controller designed using (4) for the dynamics (3) ensures that the tracking error converges to zero exponentially, and moreover, $\|w(t)\|$ is uniformly bounded in $t$ if $g<g_{\mathrm{CL}}$.

\section{B. Time-Varying External Forcing}

The method described in above extends readily to cases where the coefficient $g$ on the right hand side of (3) is time varying, i.e., $g(t)$. Notice that variable separation is not affected and we can still write the analogue of Eq. (10) and (11):

$$
\begin{gathered}
\text { Open loop : } \frac{\ddot{\gamma}-g(t) \gamma(t)}{\eta \dot{\gamma}(t)+\gamma(t)}=-\frac{\mathcal{K} \phi_{o}(x)}{\phi_{o}(x)}=-\lambda_{\mathrm{OL}}^{p} \\
\text { Closed loop : }
\end{gathered}
$$

The principal eigenvalues $\lambda_{\mathrm{OL}}^{1}$ and $\lambda_{\mathrm{CL}}^{1}$ can be found by imposing the boundary conditions on the eigenfunctions $\phi(x)$. Alternately, an approach similar to Section IV can be used to find a bound on the ratio $\lambda_{\mathrm{CL}}^{1} / \lambda_{\mathrm{OL}}^{1}$. The dynamics of $\gamma$, however, are time-varying and clearly, it is not possible to quantify the expansion of the stable envelope in the same sense as that for the time-invariant case. Nevertheless, for a given variation $g(t)$, one could use sufficient conditions such as those in [7] to compare guaranteed bounds on the stable envelope with and without the control law.

\section{CONCLUSION}

We considered a class of systems described by PDEs in which the input-output map is finite dimensional. Despite the fact that the zero dynamics are infinite dimensional, it was shown that an output regulator expands the stable operating envelope of the system by a predictable factor. The mechanism behind this expansion was shown to be the introduction of a Dirichlet boundary condition at each boundary of the system. The expansion of the stable operating envelope using just an output feedback regulator seems to be unique to systems of PDEs and without any parallel for ODE systems. The technique was demonstrated for an Euler-Bernoulli beam and extended to include output tracking controllers.

\section{ACKNOWLEDGEMENT}

Thr authors gratefully acknowledge the reviewers' comments and suggestions. The second author gratefully acknowledges support by the National Science Foundation (IIS1253758; CMMI-1427111).

\section{REFERENCES}

[1] S. Bieniawski and I. M. Kroo, "Flutter suppression using micro-trailing edge effectors," in $44^{\text {th }}$ AIAA/ASME/ASCE/AHS Structures, Structural Dynamics, and Materials Conference, 2003, AIAA 2003-1941.

[2] D. Borglund and J. Kuttenkeuler, "Active wing flutter suppression using a trailing edge flap," Journal of Fluids and Structures, vol. 16, no. 3 , pp. $271-294,2002$.

[3] R. F. Curtain and H. J. Zwart, An Introduction to Infinite-Dimensional Linear Systems Theory, ser. Texts in Applied Mathematics (Vol. 21). Springer-Verlag, 1995.

[4] M. S. P. Eastham, Q. Kong, H. Wu, and A. Zettl, "Inequalities among eigenvalues of Sturm-Liouville problems," Journal of Inequalities and Applications, vol. 3, pp. 25 - 43, 1999.

[5] G. Fix, "Asymptotic eigenvalues of Sturm-Liouville systems," Journal of Mathematical Analysis and Applications, vol. 19, pp. 519 - 525, 1967.

[6] D. H. Hodges and A. G. Pierce, Introduction to Structural Dynamics and Aeroelasticity, ser. Cambridge Aerospace Series. Cambridge University Press, 2002, no. 15.

[7] A. Ilchmann, D. H. Owens, and D. Pratzel-Wolters, "Sufficient conditions for stability of linear time-varying systems," Systems and Control Letters, vol. 9, pp. 157 - 163, 1987.

[8] M. Krstic, B.-Z. Guo, A. Balogh, and A. Smyshlyaev, "Control of a tipforce destabilized shear beam by observer-based boundary feedback," SIAM Journal of Control and Optimization, vol. 47, no. 2, pp. 553 574, 2008.

[9] A. A. Paranjape, J. Guan, S.-J. Chung, and M. Krstic, "PDE boundary control for flexible articulated wings on a robotic aircraft," IEEE Trans. Robot., vol. 29, no. 3, pp. 625 - 640, 2013.

[10] B. Ren, J. Wang, and M. Krstic, "Stabilization of an ODE Schrodinger cascade," Systems and Control Letters, vol. 62, pp. 503 $-510,2013$.

[11] G. A. Susto and M. Krstic, "Control of PDE - ODE cascades with neumann interconnections," Journal of the Franklin Institute, vol. 347, pp. $284-314,2010$.

[12] S. Tang and C. Xie, "Stabilization for a coupled PDE - ODE control system," Journal of the Franklin Institute, vol. 348, pp. 2142 - 2155, 2011.

[13] — "State and output feedback boundary control for a coupled PDE - ODE system," Systems and Control Letters, vol. 60, no. 8, pp. 540 $-545,2011$. 